\title{
Influence of short-term solar disturbances on the fair weather conduction current
}

\author{
Gal Elhalel ${ }^{1}$, Yoav Yair $^{2,5, *}$, Keri Nicoll ${ }^{4}$, Colin Price ${ }^{1}$, Yuval Reuveni ${ }^{3}$, and R. Giles Harrison ${ }^{4}$ \\ 1 Department of Geophysical, Atmospheric and Planetary Sciences, Tel-Aviv University, Israel \\ 2 Department of Life and Natural Sciences, Open University of Israel, Israel \\ ${ }^{*}$ Corresponding author: yoavya@openu.ac.il \\ 3 Institute of Geophysics and Pleasantry Physics, Scripps Institute of Oceanography, San-Diego, USA \\ 4 Department of Meteorology, University of Reading, UK \\ 5 Interdisciplinary Center Herzliya (IDC), Israel
}

Received 15 September 2013 / Accepted 6 August 2014

\begin{abstract}
The fair weather atmospheric electrical current $\left(J_{z}\right)$ couples the ionosphere to the lower atmosphere and thus provides a route by which changes in solar activity can modify processes in the lower troposphere. This paper examines the temporal variations and spectral characteristics of continuous measurements of $J_{z}$ conducted at the Wise Observatory in Mitzpe-Ramon, Israel $\left(30^{\circ} 35^{\prime} \mathrm{N}\right.$, $34^{\circ} 45^{\prime} \mathrm{E}$ ), during two large CMEs, and during periods of increased solar wind density.

Evidence is presented for the effects of geomagnetic storms and sub-storms on low latitude $J_{z}$ during two coronal mass ejections (CMEs), on 24-25th October 2011 and 7-8th March 2012, when the variability in $J_{z}$ increased by an order of magnitude compared to normal fair weather conditions. The dynamic spectrum of the increased $J_{z}$ fluctuations exhibit peaks in the Pc5 frequency range. Similar low frequency characteristics occur during periods of enhanced solar wind proton density. During the October 2011 event, the periods of increased fluctuations in $J_{z}$ lasted for $7 \mathrm{~h}$ and coincided with fluctuations of the inter-planetary magnetic field (IMF) detected by the ACE satellite. We suggest downward mapping of ionospheric electric fields as a possible mechanism for the increased fluctuations.
\end{abstract}

\section{Introduction}

The fair weather current $\left(J_{z}\right)$, which flows downward from the ionosphere through the atmosphere in fair weather regions (occurring over almost all of the Earth's area) to the Earth's surface, is a good ground-based parameter for probing the ionospheric potential (a potential difference of about $250 \mathrm{kV}$ between the ionosphere and the surface; Rycroft \& Harrison 2012). The main generators which contribute to the ionospheric potential and the Global Electric Circuit (GEC) are thunderstorms and electrified clouds, which transfer charge from cloud tops to the ionosphere. Two secondary generators are the ionospheric tides and the solar wind/magnetospheric dynamo (Roble \& Tzur 1986). Isolating the influence of one of the generators on $J_{z}$ is a complex task due to the high variability of the parameters. An additional difficulty arises from the large current variations resulting from the high sensitivity of $J_{z}$ to local conductivity changes due to variations in aerosol concentration and other local disturbances such as wind, fog and rainfall (Bennett \& Harrison 2007a)

Short-term solar disturbances such as Coronal Mass Ejections (CMEs) and Solar Energetic Particle (SEP) events, involving energetic particle precipitation and large scale ionospheric disturbances have been shown to influence $J_{z}$ and the downward pointing fair-weather electric field or Potential Gradient (PG). For instance Cobb (1967) observed an increase in PG and $J_{z}$ of $10 \%$ during periods of solar flares, compared to "quiet Sun" days, while Reiter (1989) observed much larger increases in $J_{z}$ of about $50 \%-60 \%$ following large solar flares at the Zugspitze station in the Alps. Multiple balloon launches at two different magnetic latitudes by Holzworth \& Norville (1987) have also shown an enhancement of $J_{z}$ by a factor of 2 at the southernmost balloon at $-56.3^{\circ} \mathrm{N}$ during a solar flare in 1984 . The northern balloon at $-48.8^{\circ} \mathrm{N}$ showed no response. More recently, Belova et al. (2011) analyzed measurements of the AEC (total atmospheric air-Earth electrical current) in Sweden during 35 large magnetic sub-storm events, finding an increase in the AEC about $2 \mathrm{~h}$ before $T=0$ (defined as time of minimum in $B x$ ), with an increase in the average fluctuations. Belova explained the AEC increase as enhanced $E_{z}$ at the ground due to enhancement of the ionospheric field in the (solar) storm growth and expansion phases. Another possible explanation could be changes in atmospheric conductivity $(\sigma)$ in the local $\mathrm{D}$ region due to enhanced ionization. They attributed the increased fluctuations to displacement current effects. Most evidence for solar effects on atmospheric electricity has come from high latitude regions; however, Kasatkina et al. (2009) demonstrated significant $\left(1000 \mathrm{~V} \mathrm{~m}^{-1}\right)$ perturbations of the atmospheric electric field during three ground level events (solar energetic particle events in which particles have sufficient energy to penetrate to the surface and are detected by neutron monitors) at three different zones of magnetic latitude (polar cap, auroral zone, and mid-latitude), close to the time of the causative solar flare.

Another ground-based method for studying the coupling between the magnetosphere and the mid and low atmosphere is by measurement of Ultra Low Frequency (ULF) magnetic pulsations (Kangas et al. 2001; McPherron 2005). They result from the interaction of the solar wind and Earth's magnetosphere, and are a manifestation of ULF plasma waves in the magnetosphere. The magnetic ULF pulsations occur at frequencies of $1 \mathrm{mHz}-3 \mathrm{~Hz}$ and are classified into subgroups according to their waveform; continuous or irregular, and their frequency range. The occurrence of the pulsation at a specific 
site on Earth depends on the solar wind dynamic pressure and the southward pointing magnetic flux (Sciffer et al. 2004). High latitude ionospheric electric field measurements of the pulsations are made using the Super Dual Aurora Radar Network (superDARN; Buchert et al. 1999; Chisham et al. 2007). Balloon measurements of electric ULF pulsations have also been made in the Antarctic by Bering et al. (2012).

In this paper we study the time variations and spectral characteristics of $J_{z}$ measured at Mitzpe-Ramon, Israel $\left(30^{\circ} 35^{\prime} \mathrm{N}\right.$, $34^{\circ} 45^{\prime} \mathrm{E}$ ) during two large CMEs and during periods of increased solar wind density. We present evidence for the influence of solar wind disturbances on $J_{z}$, and relate the low frequency characteristics to magnetospheric ULF waves.

\section{Instrumentation and data analysis}

$J_{z}$ is measured at Mitzpe-Ramon by the Geometrical Displacement and Conductance Current Sensor (GDACCS), which is composed of two metallic plates, one corrugated and the other flat (Fig. 1). Each plate measures the total vertical current, which is composed of contributions from $J_{z}$, the displacement current and turbulent current. The sensors' unique geometry enables $J_{z}$ to be separated from the other currents, with $J_{z}$ proportional to the difference between the currents measured by the two plates. A detailed description of the GDACCS and its operation can be found in Bennett \& Harrison (2008). The GDACCS is located at the remote Wise Astronomical Observatory near the town of Mitzpe Ramon, Israel $30.6080^{\circ} \mathrm{N}, 34.8030^{\circ} \mathrm{E}$. The sensors are placed on a flat $1.5 \mathrm{~m} \times 1.5 \mathrm{~m}$ concrete surface, so that the plates are flush with the ground, $150 \mathrm{~m}$ from the observatory dome. The signal is passed in a differential mode to the computer at the observatory, sampled at $250 \mathrm{~Hz}$ with a GPS time stamp every $1 \mathrm{~s}$.

A meteorological station, measuring the wind speed and direction, relative humidity and precipitation at a sampling frequency of $1 \mathrm{~min}^{-1}$, is located $200 \mathrm{~m}$ from the GDACCS. In addition, two magnetic induction coils and one vertical electric field ball-antenna measure the ELF/ULF signals at the site (Greenberg et al. 2007).

The raw $250 \mathrm{~Hz}$ corrugated and flat plate current values are first averaged over $1 \mathrm{~min}$ sections. The data are then divided into $30 \mathrm{~min}$ sections in which linearity between the two currents is checked, and $J_{z}$ calculated from the difference between the currents. Periods in which the $R^{2}$ value to the linear fit between the currents was less than 0.95 are not considered for further analysis. To characterize the temporal variations in $J_{z}$, the 30-min variance is calculated, which in typical fair weather conditions is $\sim 3 \mathrm{pA}^{2} \mathrm{~m}^{-4}$. The spectral analysis is performed on the raw $250 \mathrm{~Hz}$ sampled data. $J_{z}$ is calculated from the measured currents and then passed through a digital low-pass filter of $1 \mathrm{~Hz}$. The dynamic spectrum is achieved by calculating the spectrum of 30 -min sections with a 1 min time window.

\section{Observations}

This work characterizes the behavior of the vertical current at Mitzpe-Ramon (MR) throughout periods of quiet solar activity and during two different Coronal Mass Ejections (CMEs) which included Solar Proton Events (SPEs) on 24 October 2011 and 7-8 March 2012.

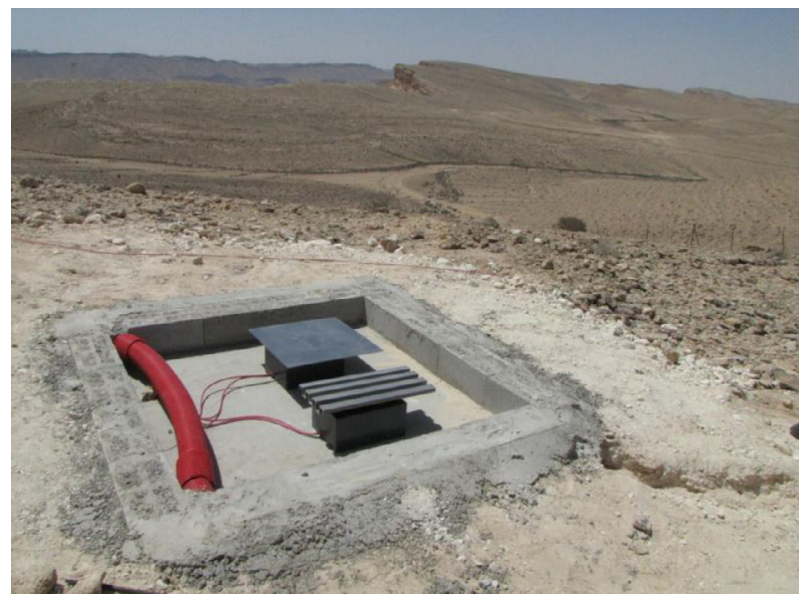

Figure 1. The GDACCS current density sensors at Mitzpe Ramon, Negev desert, Israel.

\subsection{Normal conditions: effects of local meteorology}

The Wise observatory is located in the Negev highland desert, a mountainous and arid region in southern Israel that experiences regular daily and seasonal meteorological patterns. The Mediterranean Sea breeze creates a dominant wind regime that is manifested in increasingly stronger winds from 0900 LT until 1900 LT, with a maximum around 1800 LT (Local Time, $\mathrm{LT}=\mathrm{UT}+2$ hours). Quite often, these North-Western winds are gusty and they carry dust and salt particles along their inland trajectory. The sea breeze also brings moisture that gradually increases the local relative humidity after sunset, sometimes reaching saturation and creating fog. At night, winds are much weaker and have a southeasterly component, reflecting the land breeze (Yair \& Ziv 2013).

In order to investigate the effects of solar disturbances on $J_{z}$, it is first necessary to characterize its behavior during normal, undisturbed conditions. Figure 2 shows the typical diurnal pattern of $J_{z}$, wind and relative humidity during the spring months of 2011. A clear diurnal variation is seen in $J_{z}$ (blue dots), with a maximum in the afternoon, and minimum in the early hours of the morning.

The most dominant meteorological disturbance in MR is strong wind, which from Figure 2 has a noticeable effect on $J_{z}$. The winds in MR usually reach their peak around 1600 UT and decrease in the evening, which causes an increase in the average $J_{z}$ value. During very strong wind periods $(>40 \mathrm{~km} / \mathrm{h}$ ), the individual flat and corrugated plate currents (IF and IC, respectively) are not well correlated (dashed circles in Fig. 2), possibly due to vibrations. $J_{z}$ is therefore not evaluated during these periods of strong wind. A detailed description of the influence of the wind speed on $J_{z}$ is given in Appendix.

The GDACCS measures the charge flowing downward to the plates. Therefore, positive charge flowing downward should be measured as positive current. The measured current in MR is of the expected magnitude ( $\mathrm{a}$ few $\mathrm{pA} / \mathrm{m}^{2}$ ), but negative. In order to resolve the disparity we have placed an identical system a few meters away. Both systems measured negative values and showed a very good agreement between measured currents and their fluctuations.

Measurements made from a different desert site in Israel resulted in positive values and presented the same increase with wind speed. These results imply that the negative values probably result from a small offset due to geographical 


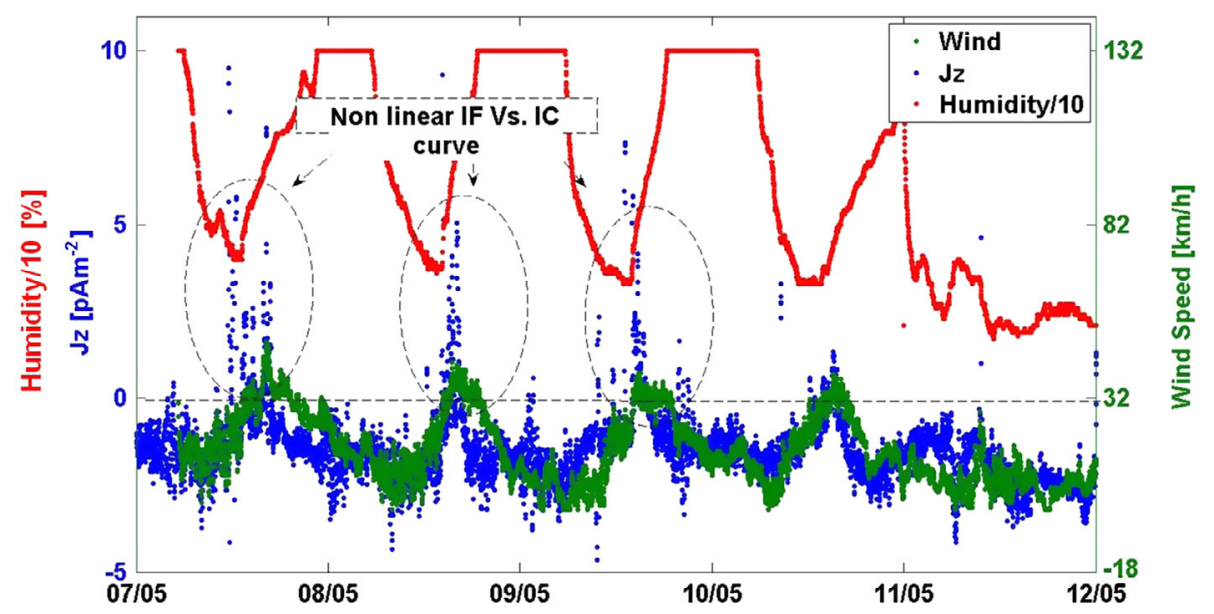

Figure 2. A week of data of $J_{z}$ values (blue points) from 7 May 2011 to 12 May 2011. Daily variations are superimposed on the changes in wind speed (green) and relative humidity (red). Dashed horizontal line indicates zero current.

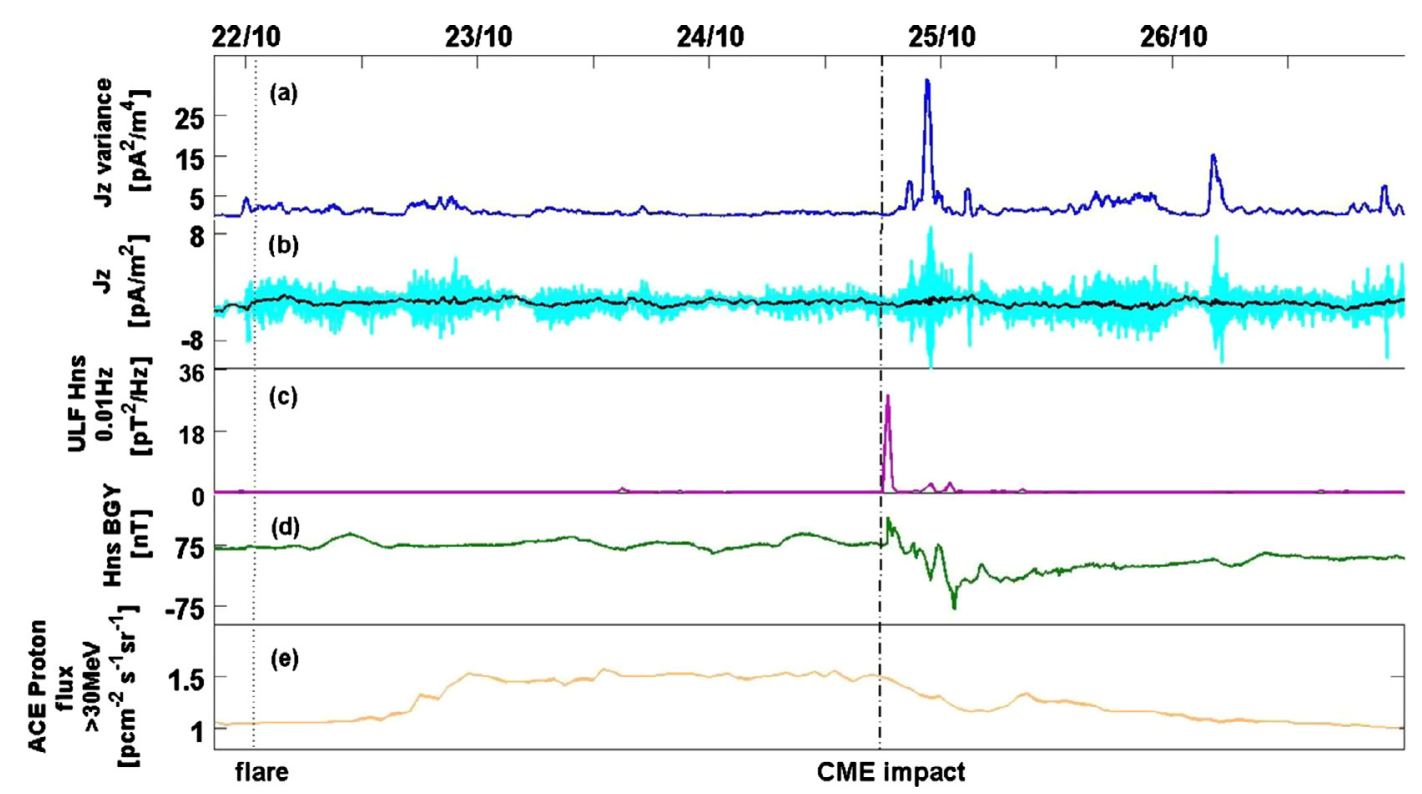

Figure 3. (a) $J_{z}$ fluctuations measured in MR during 22-27 of October 2011. (b) Average $J_{z}$ (turquoise) and the 30 min averaged $J_{z}$ (black). (c) Magnetic ULF spectrum at $0.01 \mathrm{~Hz}$ measured in MR. (d) Magnetic field NS DC component, Bar Giora, Israel. (e) ACE $30 \mathrm{MeV}$ Proton Flux. Dotted vertical line marks the flare onset. Dashed vertical line marks the CME impact with the atmosphere.

distortion of the potential gradient in the mountainous landscape of the site.

For this reason, this work discusses only the current fluctuations and not the current magnitude and sign. The average magnitude is used only for excluding disturbed weather periods and as a reference value for normal condition measurements.

\section{2. $J_{z}$ fluctuations in conjunction with $\mathrm{CME}$ impacts}

\subsubsection{4-25 October 2011}

On 22nd October at 0058 UT an M-class flare released a significant CME which hit Earth's magnetic field on 24th October at 1748 UT (Fig. 3). The ACE spacecraft measured an increased high energy proton flux $(>30 \mathrm{MeV})$ on the 22 nd around 1800 UT, associated with the flare, which lasted until the early morning of the 25th (Fig. 3e). The CME caused a strong compression of Earth's magnetic field (Fig. 3d), with the $K$ index reaching a maximum of 9 on the 24th around 2300 UT. The Dst value (Disturbance storm time, which denotes the magnitude of the disturbance in the horizontal geomagnetic field in equatorial and mid latitudes) showed a decrease of $-120 \mathrm{nT}$ in the early hours of 25 th October (WDC for Geomagnetism, Kyoto).

The response detected at MR is shown in Figures $3 a-3 c$. The measured $J_{z}$ exhibits a large increase in fluctuations on 24th October around 2300 UT (Fig. 3a, blue curve), an order of magnitude larger than the typical fair weather fluctuations. The large fluctuations lasted for a duration of $7 \mathrm{~h}$. Figure $3 \mathrm{c}$ (purple curve) shows the amplitude of the magnetic ULF spectrum at $0.01 \mathrm{~Hz}$ also measured at MR. A significant fluctuation in the ULF spectrum is present at 1823 UT which coincides with variability in the North-South component of the geomagnetic field measured at the Bar Giora Observatory $\left(31^{\circ} 43^{\prime} \mathrm{N}, 35^{\circ} 5^{\prime} \mathrm{E}\right)$, Israel (Fig. 3d). The onset of $J_{z}$ 


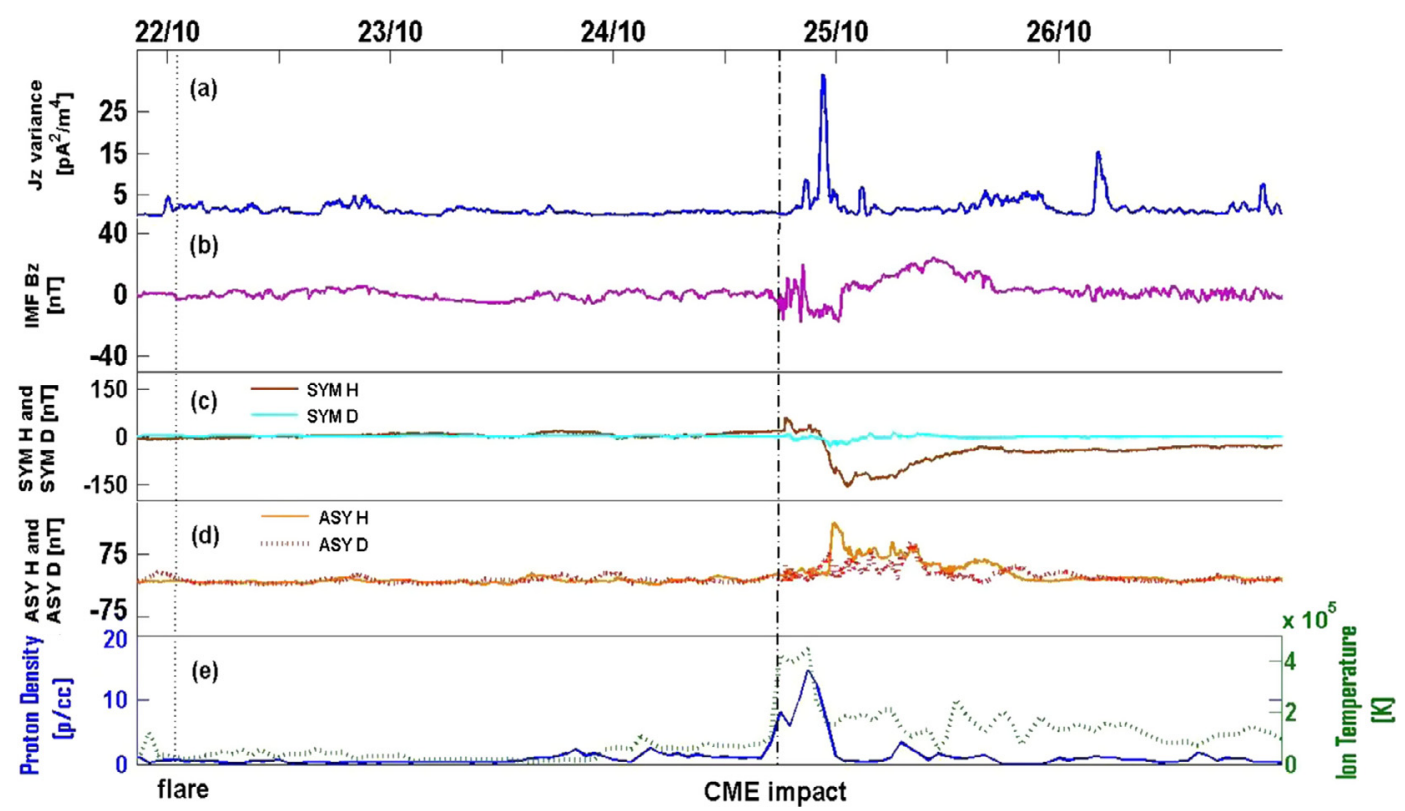

Figure 4. (a) $J_{z}$ fluctuations measured in MR during 22-27 of October 2011; (b) IMF $B_{z}$; (c) The horizontal symmetric geomagnetic disturbance field (SYM H, brown curve) and the orthogonal symmetric geomagnetic disturbance field (SYM D, turquoise curve); (d) The horizontal asymmetric geomagnetic disturbance field (ASY H, orange curve) and the orthogonal asymmetric (ASY D, red dotted curve); (e) ACE SW proton density (blue curve) and SW ion temperature (green dotted curve).

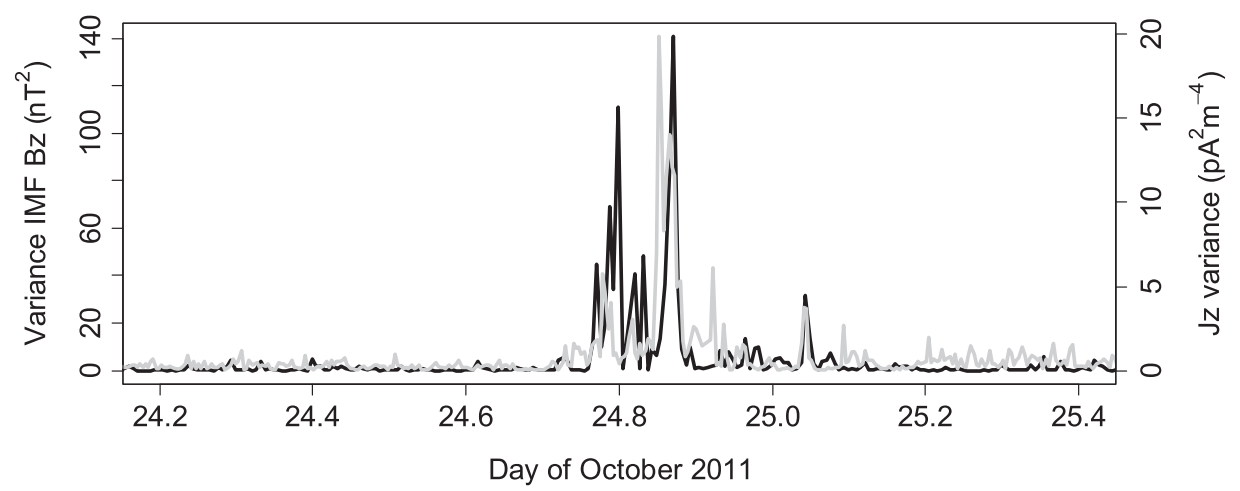

Figure 5. Time series of variance in $J_{z}$ (5-min values) (gray line) from MR on 24th and 25th October 2011, plotted alongside variance in $z$ component of interplanetary magnetic field (IMF $B_{z}$ ) measured by the ACE spacecraft. $B_{z}$ time data was corrected to account for propagation time from the location of the ACE spacecraft to Earth (using a distance of $1.5 \times 10^{6} \mathrm{~km}$ (L1 point) and the observed solar wind speed).

fluctuations occurred $2 \mathrm{~h}$ after the rise in the magnetic ULF spectrum and the Bar-Giora DC component. This time lag indicates that the fluctuations did not result from magnetically induced currents in the cables. The ULF/ELF station is located less than $100 \mathrm{~m}$ from the $J_{z}$ system, and connected to the same monitoring PC. During the exact time of the fluctuations in $J_{z}$ there was no evidence for changes in the ELF and ULF data. Figure 4 presents the SWEPAM solar wind parameters $(4 \mathrm{e})$ and the geomagnetic $(4 \mathrm{c}, 4 \mathrm{~d}$, WDC for Geomagnetism in KYOTO) and interplanetary (4b) magnetic field parameters during the October 2011 event. The $J_{z}$ fluctuations occurred during periods of mid-latitude geomagnetic disturbances (Figs. 4c and 4d), and when the proton density is high.

Figure 5 examines the changes in $J_{z}$ on 24th October in more detail. The gray line denotes the 5 -min variance in $J_{z}$, and the black line, the variance in the $z$ component of the interplanetary magnetic field (IMF) measured by the ACE satellite. There is a striking similarity between the two, suggesting firstly, that that the $J_{z}$ fluctuations at MR were not a result of fluctuations in local meteorological conditions, and secondly that the $J_{z}$ variations are directly related to changes in the magnetic field of the solar wind.

\subsubsection{7-8 March 2012}

The period surrounding 5th-8th March 2012 consisted of several energetic solar events, the largest two comprising an X1/2 flare emitted at 0409 UT on 5th March, and an X5/3 flare emitted at 0024 UT on 7th March, both with Earthward directed CMEs. The CME from the X1/2 flare impacted Earth's magnetosphere at 0427 UT on 7th March and the impact from the X3/5 flare associated CME occurred at 1105 UT on 8th March. The 8th March 2012 event was significantly stronger than the October 2011 event and was also accompanied by an increase in the flux of energetic protons, with energy higher than $100 \mathrm{MeV}$, starting at $0405 \mathrm{UT}$ on 7th March, associated with 
G. Elhalel et al.: Effects of solar disturbances on fair weather current

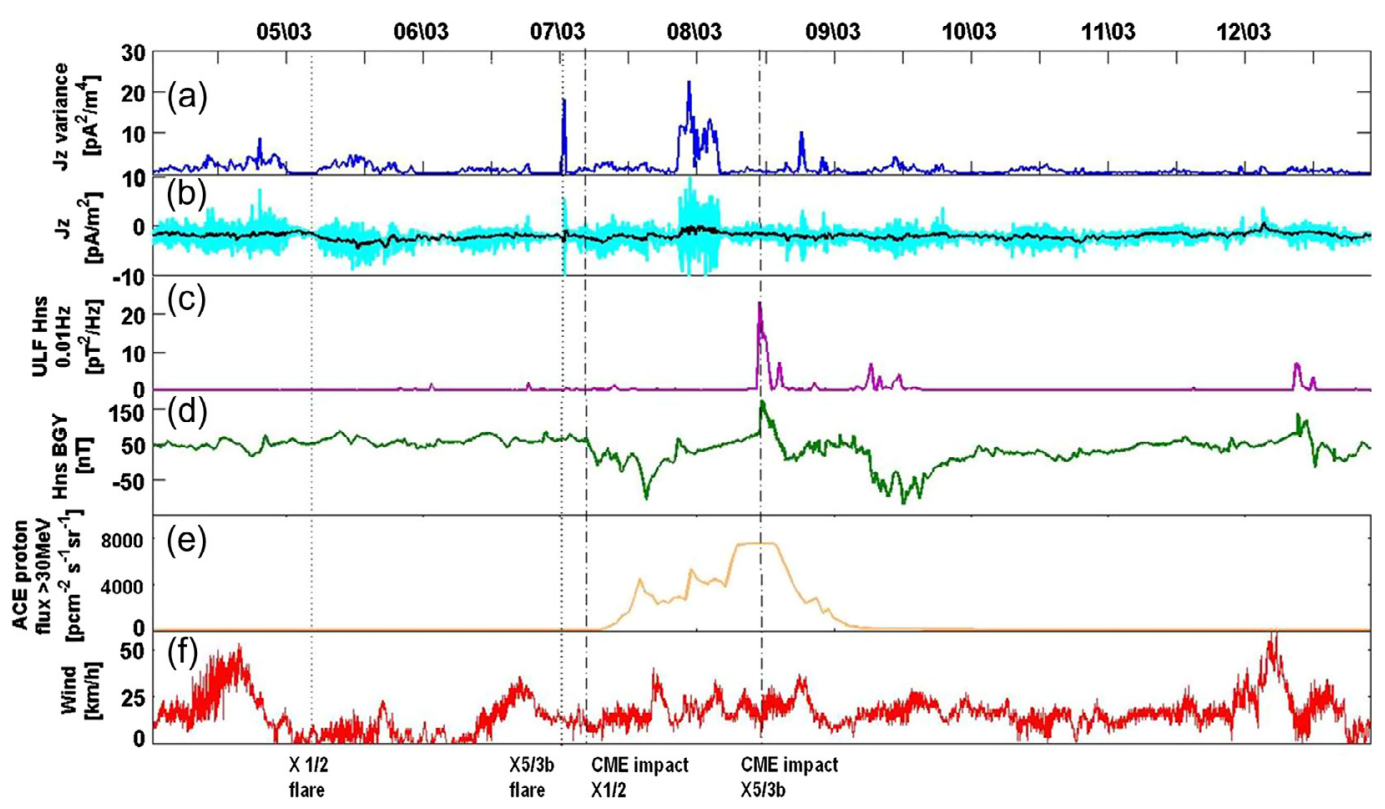

Figure 6. (a-e) The same as Figure 3, for 7-8th March 2012, event. (f) Wind speed.

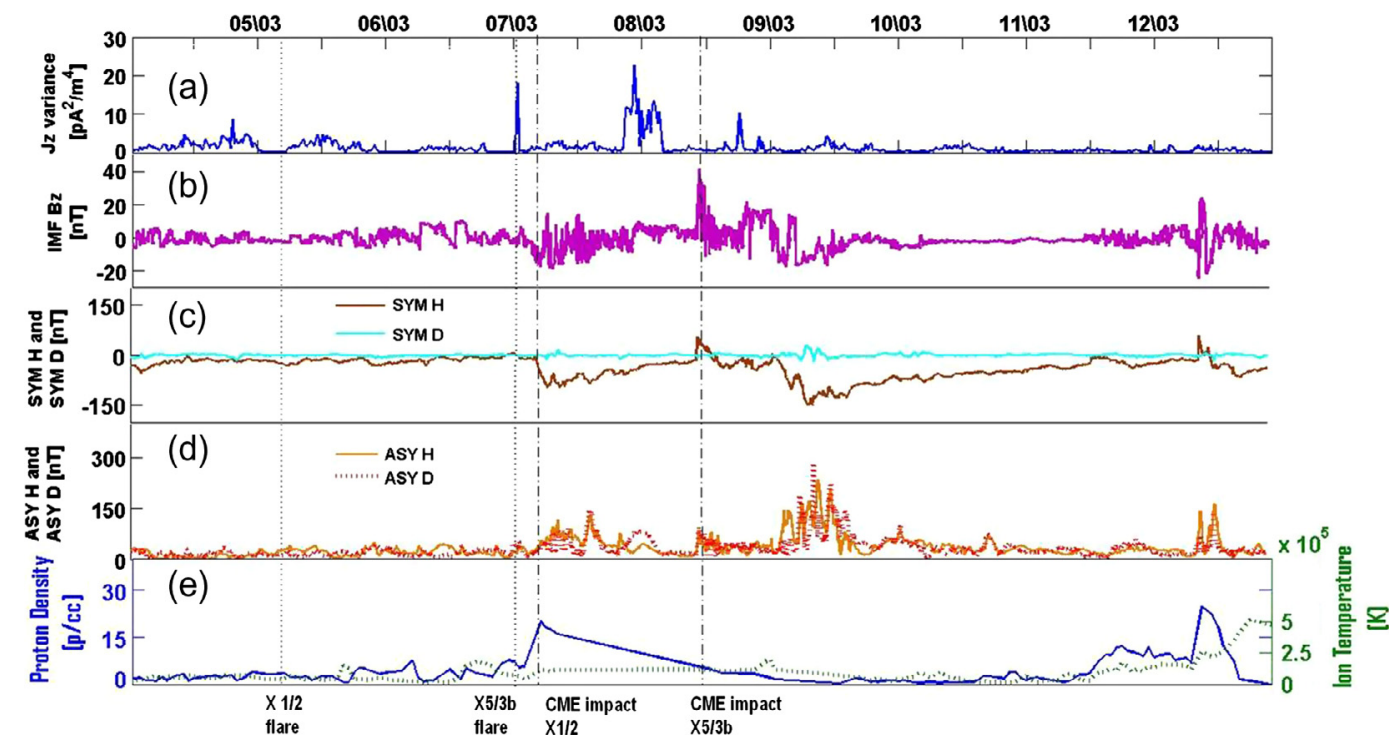

Figure 7. Same as in Figure 4, for 7-8th March 2012, event.

the $\mathrm{X} 5 / 3$ flare. This event is now considered one of the strongest events in the present solar cycle, with a solar proton flux of energetic protons $(>10 \mathrm{MeV})$ in excess of 6500 proton flux units. The Dst index shows two (negative) peaks, on the 7 th early morning hours $(-70 \mathrm{nT})$ and the 9th $(-110 \mathrm{nT})$ and thus indicates a decrease in the horizontal geomagnetic field strength.

Figure 6 shows data for the March 2012 event, as for Figure 3. Similar to the October 2011 event, $J_{z}$ showed significant fluctuations, much larger than normal fair weather values intermittently from 7th to 8th March 2012 (Fig. 6a). The increase in the low frequency ULF spectrum (Fig. 6c) occurred $6 \mathrm{~h}$ after the $J_{z}$ fluctuations ceased. There was no unusual behavior in the wind speed and humidity measurements at the Wise observatory during the period from 7th to 8th March (Fig. 6f). Figure 7 presents the SWEPAM solar wind parameters (7e) and the geomagnetic (7c, 7d, WDC for Geomagnetism in KYOTO) and interplanetary (7b) magnetic field parameters during the March event.

\subsubsection{Spectral characteristics}

Examination of the dynamic spectrum (calculated from the $1 \mathrm{~Hz}$ low-pass filtered data, on 30-min sections with a 1-min time window) of the measured $J_{z}$ fluctuations revealed peaks in the $\mathrm{mHz}$ range $(2-8 \mathrm{mHz})$ during the time of the increased $J_{z}$ fluctuations for both of the solar events (Fig. 8). Figures 8a and $8 \mathrm{~b}$ display the dynamic spectrum of $J_{z}$, measured on the 24 October 2011 and 08 March 2012, respectively. The red curve in the inset shows a section of the increased fluctuations in the filtered data (red curve, left inset) and an example for a 30-min spectrum from this section (green curve, right inset). This frequency range coincides with the ULF Pc5 pulsations frequencies. 

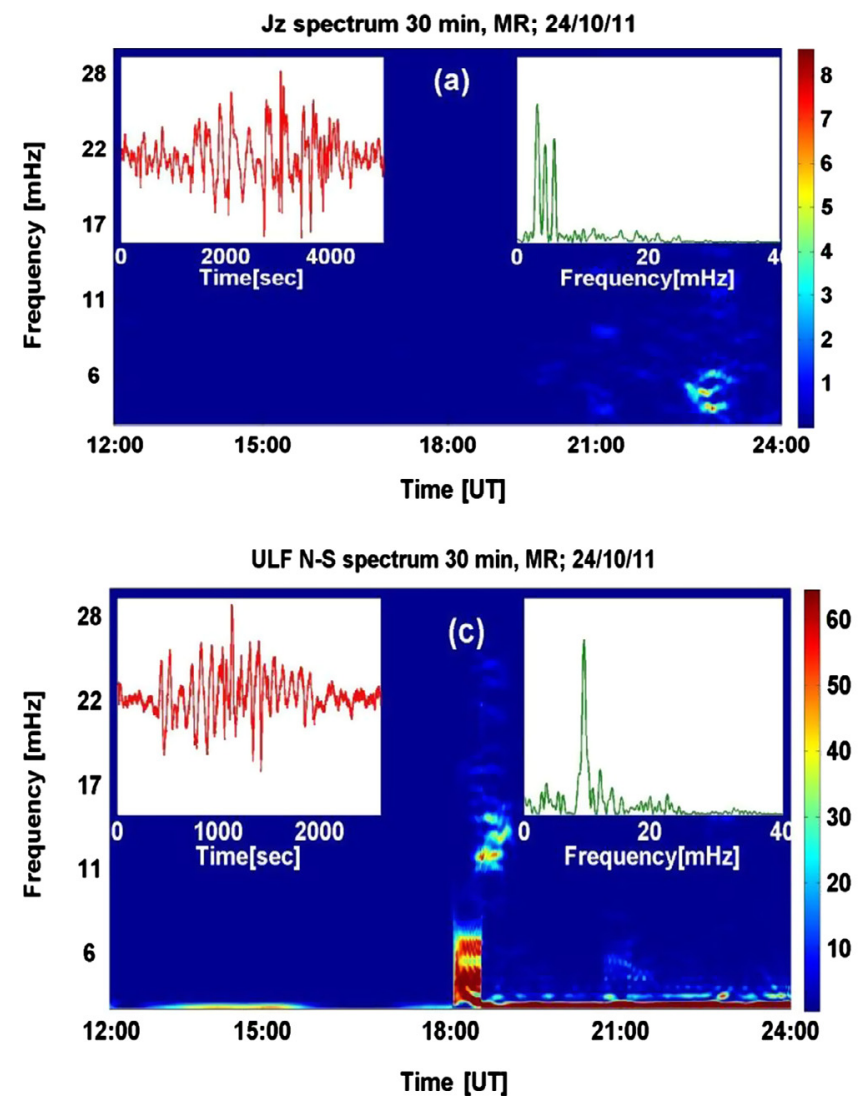
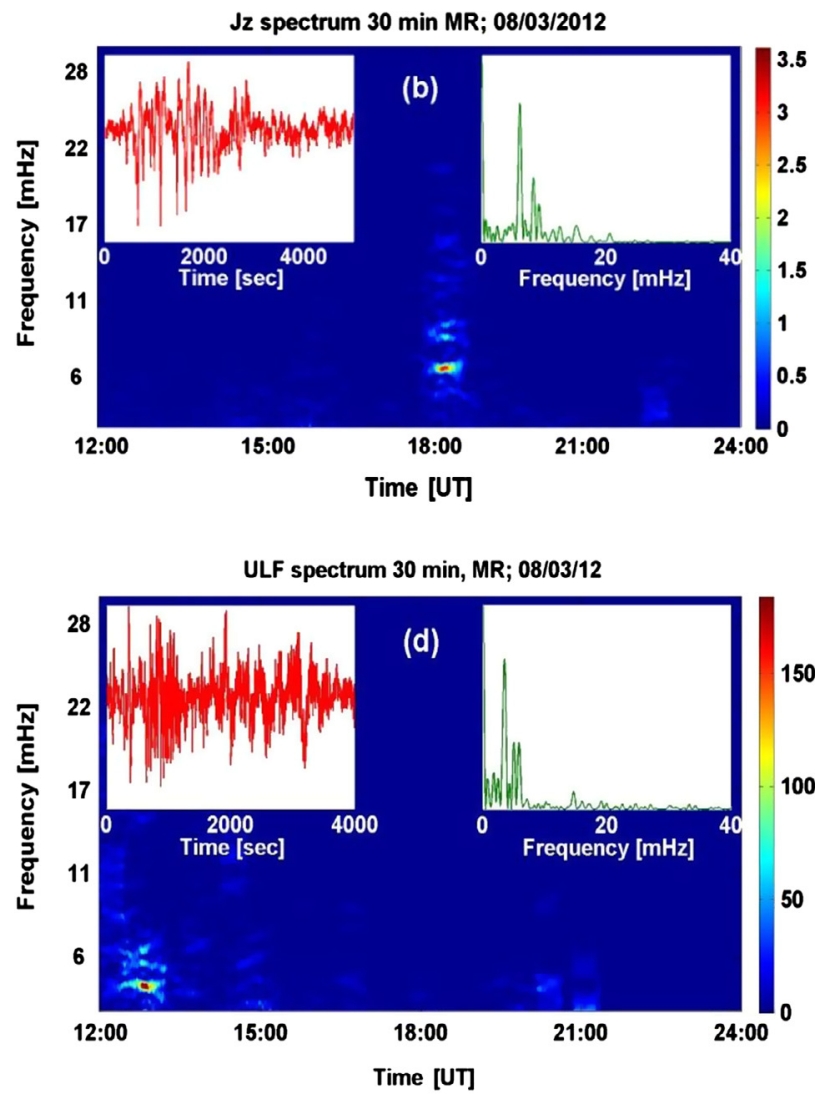

Figure 8. (a) $J_{z}$ dynamic spectrum 24 October 2011. (b) $J_{z}$ dynamic spectrum 08 March 2012. (c) ULF Hns dynamic spectrum 24 October 2011. (d) ULF Hns dynamic spectrum 8 March 2012. Color bars for $J_{z}$ are in $\mathrm{pA} \mathrm{m}^{-2} / \sqrt{ } \mathrm{Hz}$ and $\mathrm{pT}^{2} / \mathrm{Hz}$ for the ULF spectrum.

Figures $8 \mathrm{c}$ and $8 \mathrm{~d}$ show the horizontal magnetic ULF dynamic spectrum measured at the same site. For the October event, the magnetic field spectrum exhibits a peak at a higher frequency $(\sim 10 \mathrm{mHz}) 4 \mathrm{~h}$ before the $J_{z}$ frequency peak, and only a very weak signal at the time of the $J_{z}$ fluctuations at a higher frequency. For the March event the magnetic low frequency peak appears more than $5 \mathrm{~h}$ before the $J_{z}$ peak at a lower frequency.

\subsection{Long-term measurements}

In contrast to the $J_{z}$ measurements, the low frequency $J_{z}$ spectrum does not exhibit diurnal variations, and is not highly influenced by the wind speed and relative humidity. As a result, relatively weak signals can be detected, which are not masked by the local atmospheric noise.

The dynamic spectrum of $J_{z}$ was therefore examined for four continuous months of measurements (March-June 2012) (Fig. 9). Low frequency peaks during the early mornings and late evenings when wind speed was low were searched for, and only on days for which the individual flat and corrugated plate currents were correlated throughout the day. During this period (99 fair weather days), low frequency $J_{z}$ peaks were found to occur on 19 days. Fourteen of these peaks occurred on days when the solar wind proton density was higher than $5 \mathrm{pcm}^{-3}$ (Fig. 9). Most peaks occurred during local dusk or dawn. Five peaks occurred on quiet days, when the solar wind proton density was low. There was no correlation with the solar wind speed. The two days with the highest proton density (12 March 2012 and 16 June 2012) showed no low frequency peaks in $J_{z}$.

\section{Discussion}

The results presented here show two distinct features of the vertical current behavior during periods of increased solar activity - an increase in the $J_{z}$ variability during the two large CME events; and an ultra-low frequency peak in the dynamic spectrum, observed both in the large CME events and also in the more frequent periods of high solar wind proton density. When searching for the mechanism responsible for the phenomena one must consider two scenarios. One, in which the two features are of the same origin, and the increased variability seen during the large CME events is the manifestation of a large increase in low frequency fluctuations. The alternative is that two or more separate mechanisms are involved.

\subsection{Local meteorological influences on $J_{z}$}

As is evident from the results presented in Section 3.1, the vertical current is highly sensitive to meteorological factors such as wind speed (Ette 1972) and local aerosol number concentration (Bennett \& Harrison 2007a). It is therefore necessary to preclude the influence of local sources on $J_{z}$. Unfortunately, no meteorological data were available during the October 2011 event due to a system malfunction, however there are several factors which support the conclusion that the fluctuations observed in $J_{z}$ on 24th-25th October were not of a local meteorological origin. One is that the variability in $J_{z}$ increases during the late evening of the 24th, a time at which the wind speed usually decreases and becomes calm during the night. Further, the average value of $J_{z}$ was fairly constant, therefore it is unlikely that the fluctuations resulted from variations in wind speed or direction. Very strong winds can be easily ruled out due to 


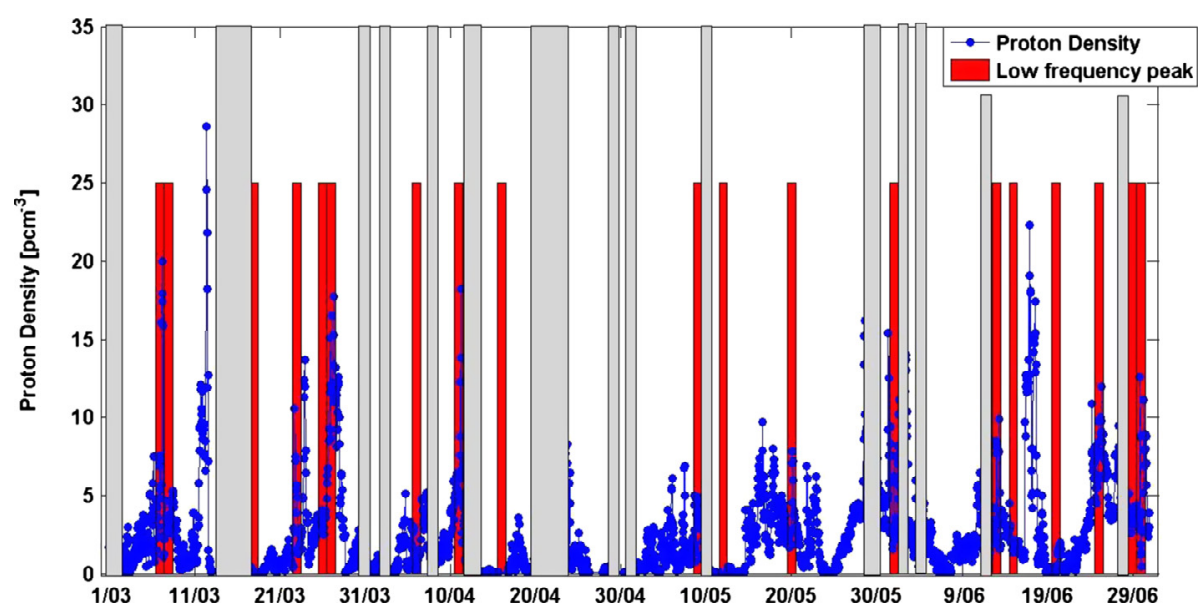

Figure 9. Occurrence of low frequency $J_{z}$ peak plotted alongside ACE solar wind proton density. Red bars - days with low frequency peak, blue points - ACE solar wind proton density measurements. Gray rectangles mark non fair weather periods.

the very close linear relationship $\left(R^{2}>0.999\right)$ between the flat and corrugated plate currents, which does not hold when the wind speed is greater than $45 \mathrm{~km} / \mathrm{h}$. Further evidence that the $J_{z}$ fluctuations were not caused by local meteorological variations is shown in Figure 5, which demonstrates a close correlation between the $J_{z}$ fluctuations and variability in the IMF $B_{z}$ component.

The 7-8th March 2012 event occurred around midnight, when humidity and wind speed were low (Fig. 6f), therefore it is unlikely that the increased $J_{z}$ fluctuations were caused by local meteorological influences. The long-term spectral analysis was performed only for early mornings and late evenings when wind speed was low, and only on days for which the individual flat and corrugated plate currents showed a clear linear relationship between each other throughout the day, in order to diminish the local meteorological noise.

Further evidence in support of a solar effect on $J_{z}$ is that during the two CME events the measured $J_{z}$ fluctuations were an order of magnitude larger than the typical fair weather values. This implies an additional mechanism which contributes to the increased $J_{z}$ fluctuations, other than local meteorological effects alone.

\subsection{Energetic particle and displacement current effects}

The magnitude, the oscillatory nature and short timescale of the $J_{z}$ fluctuations suggests that the source of the changes were displacement currents (caused by changing electric fields) rather than conduction effects. The GDACCS was designed to distinguish between displacement and conduction currents, by measuring the same displacement current, but different conduction currents, through a difference in cross sectional area between the two plates. Although the method of $J_{z}$ calculation (found by subtracting the difference in total current measured by the two plates) should remove any effect of displacement currents, it is possible that geometrical distortions caused by the arrangement of the plates could give rise to unequal displacement currents. This therefore means that the cancellation of displacement currents using the two plates may not be complete, and therefore we cannot preclude the impact of electric field variations causing the observed $J_{z}$ fluctuations.

There are two likely sources of electric field changes, and thus displacement currents, which can occur during active solar events - enhanced ionization from energetic particle precipitation, and downward mapping of ionospheric electric field changes. Figures $3 \mathrm{e}$ and $4 \mathrm{e}$ demonstrate that both the October 2011 and March 2012 events were associated with SEP events, which would increase the flux of energetic particles entering Earth's atmosphere and therefore enhance ionization. SEPs have the largest effect at high geomagnetic altitudes due to reduced shielding around the geomagnetic poles, where high energy particles can easily penetrate Earth's magnetic field. The low geomagnetic latitude of the measurement site (with geomagnetic cutoff rigidity $\sim 11 \mathrm{GV}$ ) suggests that only extremely energetic particles can penetrate and ionize the atmospheric column above Israel, therefore the $J_{z}$ fluctuations are unlikely to be directly associated with enhanced ionization from the SEP events. Despite this, recent work has shown that substantial displacement currents associated with even relatively weak SEP events can arise in atmospheric electricity measurements at mid-latitudes. Nicoll \& Harrison (2014) demonstrate fluctuations in $J_{z}$ (measured using a GDACCS instrument) and $E_{z}$ in the UK during a relatively weak solar proton event in 2013. The fluctuations are thought to be associated with variations in the ionization rate above the UK (measured simultaneously from a balloon, but not detected by surface neutron monitors), which perturb the electric field in the lower troposphere sufficiently to induce displacement currents at the surface. Furthermore, electric field changes of $1000 \mathrm{~V} \mathrm{~m}^{-1}$ during a 10 min period were reported by Kasatkina et al. (2009) at high latitudes $(63.3 \mathrm{~N})$ during three ground level events in 2001. The variations spread over a large area and reached down to mid-latitudes $\left(50 \mathrm{~V} \mathrm{~m}^{-1}\right.$ changes in $E_{z}$ at $\left.47.2 \mathrm{~N}\right)$. The proton flux measured during these events was similar and even smaller than the 8 March 2012 event described here, therefore even though it is unlikely, the possibility that highly energetic particles were able to penetrate to the latitude of Israel exists.

Downward mapping of horizontal changes in ionospheric potential has been suggested as a method of coupling between ionospheric potential changes and the atmospheric electric field at the surface. Park (1976) calculated the surface vertical electric field induced by a horizontal ionospheric potential difference, demonstrating that the vertical electric field variations near the ground follow the horizontal potential differences in the ionosphere. The magnitude of the surface electric field depends on the near earth conductivity. For a displacement current of $10 \mathrm{pA} \mathrm{m}^{-2}$ at frequency of $5 \mathrm{mHz}$, the inductive 
surface electric field should be of the order of $100 \mathrm{~V} \mathrm{~m}^{-1}$. A very large $(\sim 5000 \mathrm{~km})$ ionospheric disturbance of $50 \mathrm{mV} \mathrm{m}^{-1}$ would result in a surface electric field change of this scale (similar magnitude to electric field measured by Kasatkina et al. 2009) which could induce currents of similar magnitudes to those observed in the Mitzpe Ramon $J_{z}$ measurements. It is likely that such large ionospheric disturbances can arise in the hours following a CME, however, effects are generally expected to be confined to high latitude regions. The results reported here thus present an interesting situation such that if source of the $J_{z}$ fluctuations is downward mapping of ionospheric potential changes, then this would seemingly affect much lower geomagnetic latitudes than previously thought.

\subsection{ULF considerations}

Another vindication for the displacement current explanation is the fluctuating nature of the disturbance and the dominant ULF frequency peak. Magnetospheric ULF electric $\left(\mathrm{mV} \mathrm{m}^{-1}\right)$ and magnetic (nT) fields generated by the interaction of the solar wind with the magnetosphere are common during periods of high solar wind and geomagnetic storms (Liu et al. 2009). The ionospheric response to these magnetospheric disturbances is complex and depends on variable factors such as the conductivity profile, local time, and the dip angle (Glassmeier \& Stellmacher 2000; Sciffer et al. 2004). The induced currents in the ionosphere modify the waves' amplitude and spatial scale size and a further attenuation occurs through the interaction with the atmosphere.

Very intense $(\sim 500 \mathrm{nT})$ magnetic pulsations in the Pc5 range $(2-7 \mathrm{mHz})$ have previously been observed during severe magnetic storms (Kleimenova \& Kozyreva 2005). Pulsations with identical frequency spectra and polarization and with close amplitudes were observed in a very wide range of geomagnetic latitudes (from $60^{\circ}$ to $70^{\circ}$ ). According to Park (1976) such a large and coherent ionospheric disturbance can be mapped down to vertical electric fields at the surface, of the appropriate magnitude to induce the observed low frequency fluctuations of $J_{z}$ during the two large CME events.

The absence of a simultaneous magnetic ULF signal could result from ionospheric shielding, where Faraday inductive currents reduce the amplitude of the magnetic waves measured on the ground. The magnetic pulsations measured on a different time that day, with the higher frequency peaks were probably of a different origin.

Only $73 \%$ of the weaker low frequency transients observed in the long-term measurements occurred during periods of increased solar wind density. The maximum peak amplitude showed no correlation with the solar wind parameters. A more thorough examination of the ionospheric characteristics such as the dip angle, local time and conductivity profile is needed for a proper comparison. The distance to the disturbance center should also be taken into account.

\section{Summary and conclusions}

This paper examines the behavior of the vertical conduction current measured at Mitzpe-Ramon, Israel during periods of increased solar activity. During the two large CME events studied, variability in $J_{z}$ was observed, which cannot be attributed to meteorological factors. A low frequency peak in the dynamic spectrum was also observed, which is present in both the large CME events and also during frequent periods of high solar wind proton density, analyzed separately. The low frequency peaks are in the Pc5 frequency range. The increased variability in $J_{z}$ during the large CMEs is an order of magnitude larger than the typical fair weather values. For the October 2011 event, the $J_{z}$ fluctuations coincided with fluctuations of the IMF. It is not yet clear whether the increased fluctuations during the large CMEs and the low frequency peaks are of the same origin and if one or more mechanisms are responsible for the observed characteristics. Large scale $(\sim 5000 \mathrm{~km})$ ionospheric fluctuating disturbances mapped to vertical surface electric field may result in vertical current fluctuations of the same order of the measured current fluctuations. We therefore suggest downward mapping of ionospheric electric fields as a possible mechanism for the increased fluctuations; however, due to the large variety of processes involved in these large solar events it is difficult to determine the exact source of the increased fluctuations. Simultaneous measurements of $J_{z}, \mathrm{PG}$, and magnetic ULF pulsations during large solar events and high solar wind proton flux periods, and comparison to ionospheric electric field measurements and ionization rates, are needed in order to fully understand these results and will be the subject of future study.

Acknowledgements. The authors would like to thank the Wise Observatory, and to Boris Shirman from Israel Survey for supplying Bar Giora magnetic DC data. ACE IMP, proton flux and solar wind proton density data was obtained from NOAA SWPC. KAN acknowledges an Early Career Fellowship from the Leverhulme Trust. This research was supported by the Israeli Science Foundation (Grant No. 423/14). The editor thanks Michael Rycroft and two anonymous referees for their assistance in evaluating this paper.

\section{Appendix}

To further investigate the relationship between $J_{z}$ and wind speed, Figure A1-a presents the relative change of $J_{z}$ and wind speed measured around noon, when humidity is low, and the wind speed is relatively high. There is a very good correlation between the two variables. Further, Figure A1-b demonstrates the strong linear relationship between $J_{z}$ and wind speed (using values across the whole day) for large wind speeds (greater than $15 \mathrm{~km} / \mathrm{h}$ ). Using the slope of the linear fit, $J_{z}$ can be estimated from the wind speed (red line in Fig. A1-b) and compared to the measured $J_{z}$ (blue points). The linear dependency of the vertical current on wind speed has been explained by Ette as due to advection of space charge (Ette 1972). Ette demonstrated that the horizontal wind contribution to the current $\left(J_{\text {adv }}\right)$ is proportional to the wind speed $\left(v_{x}\right)$, and its direction depends on the space charge density $(\rho)$ and effective electric susceptibility:

$$
J_{\mathrm{adv}} \sim v_{x} \frac{\partial E_{z}}{\partial x} \sim \frac{\partial}{\partial x} \int_{0}^{z} \rho_{z} \mathrm{~d} z
$$

Ette related the advection contribution to wind speeds higher than $\sim 20 \mathrm{~km} / \mathrm{h}$, and expected an addition of $\sim 1 \mathrm{pA} / \mathrm{m}^{2}$ for a wind speed greater than $35 \mathrm{~km} / \mathrm{h}$. Figure A1-b demonstrates the similarity between the relationship derived by Ette (1972), where the ratio between $J_{z}$ and the wind speed was 0.67 , and the ratio of 0.733 measured at MR. 
(a)

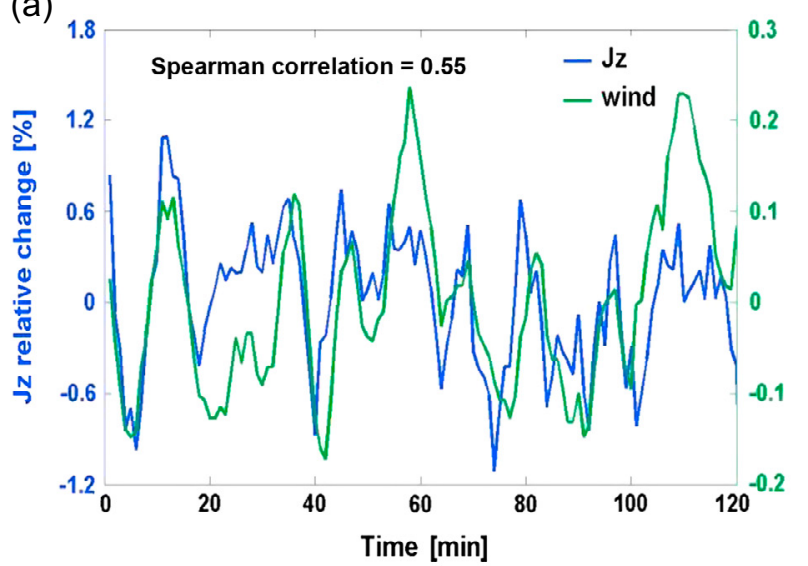

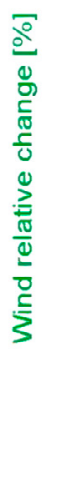

(b)

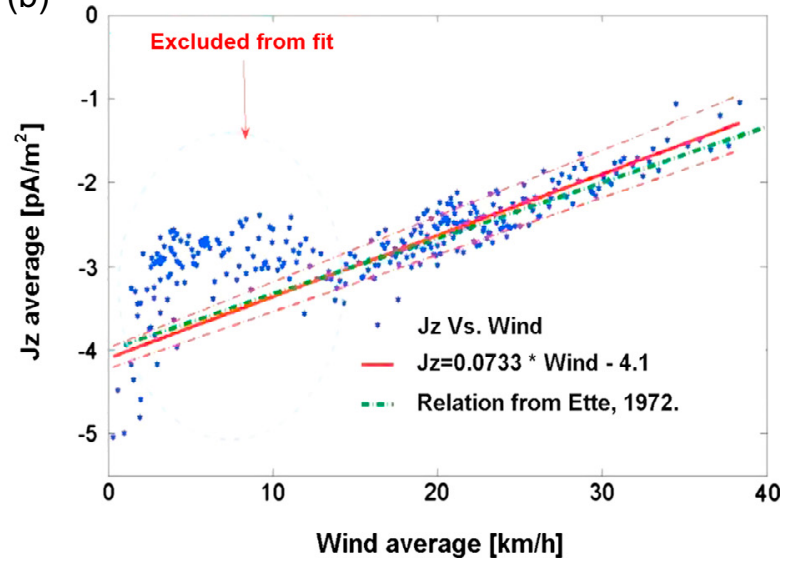

Figure A1. Correlation between wind speed and Jz. (a) Relative changes of $J_{z}$ and wind speed from 2 h of measurements taken on the 10 May 2011. (b) $J_{z}$ vs. wind speed. Blue dots - measured data; Red solid line - linear fit to measured data. Green line - Ette's linear relation.

\section{References}

Belova, E., S. Kirkwood, and H. Tammet, The effect of magnetic substorms on near ground atmospheric current, Ann. Geophys., 18, 1623-1629, DOI: 10.1007/s00585-001-1623-z, 2011.

Bennett, A.J., and R.G. Harrison, Variability in surface atmospheric electric field measurements, J. Phys: Conf. Ser, 142, DOI: 10.1088/1742-6596/142/1/012046, 2007a.

Bennett, A.J., and R.G. Harrison, Atmospheric electricity in different weather conditions, Weather, 62, 227-283, DOI: 10.1002/wea.97, $2007 b$.

Bennett, A.J., and R.G. Harrison, Surface measurement system for the atmospheric electrical vertical conduction current density, with displacement current density correction, J. Atmos. Sol. Terr. Phys., 70, 1373-1381, DOI: 10.1016/j.jastp.2008.04.014, 2008.

Bering, E.A., J.R. Benbrook, M.J. Engebretson, and R.L. Arnoldy, Simultaneous electric and magnetic field observations of Pc1-2, and Pc3 pulsations, J. Geophys. Res, 103, 6741-6761, DOI: 10.1029/97JA03327, 2012.

Chisham, G., M. Lester, S.E. Milan, M.P. Freeman, W.A. Bristow, et al., A decade of the Super Dual Auroral Radar Network (SuperDARN), Surv. Geophys., 28, 33-109, DOI: 10.1007/s10712-007-9017-8, 2007.

Cobb, W.E., Evidence of a solar influence on the atmospheric electric elements, Mon. Weather Rev., 95, 905-911, 1967.

Ette, A.I., An effect of space charge advection on vertical air-Earth current measurements, Arch. Met. Geoph. Biokl. Ser. A, 21, 329-338, DOI: 10.1007/BF02247981, 1972.

Glassmeier, K.-H., and M. Stellmacher, Concerning the local time asymmetry of PC5 power at the ground and field line resonance width, J. Geophys. Res., 105, A8, DOI: 10.1029/2000JA900037, 2000.

Greenberg, E., C. Price, Y. Yair, M. Ganot, J. Bor, and G. Satori, ELF transients associated with sprites and ELVES in eastern Mediterranean winter thunderstroms, J. Atmos. Sol. Terr. Phys., 69, 1569-1586, 2007.

Holzworth, R.H., and K.W. Norville, Solar flare perturbations in stratospheric current systems, Geophys. Res. Lett., 14, 852-855, DOI: 10.1029/GL014i008p00852, 1987.
Kangas, J., J. Kultima, A. Guglielmi, A. Potapov, and K. Hayashi, Impact of interplanetary shock on the ULF wave activity: A case study of the storm sudden commencement on September 22, 1999, Earth Planet Space, 53, 1177-1182, 2001.

Kasatkina, E.A., O.I. Shumilov, M.J. Rycroft, F. Marcz, and A.V. Frank-Kamenetsky, Atmospheric electric field anomalies associated with solar flare/coronal mass ejection events and solar energetic charged particle "Ground Level Events, Atmos. Chem. Phys., 9, 21941-21958, DOI: 10.5194/acpd-9-21941-2009, 2009.

Kleimenova, N.G., and O.V. Kozyreva, Spatial-Temporal Dynamics of Pi3 and Pc5 Geomagnetic Pulsations during the Extreme Magnetic Storms in October 2003, Geomag. Aeron., 45, 75-83, DOI: 10.1029/2011JA016931, 2005.

Liu, W., T.E. Sarris, X. Li, S.R. Elkington, R. Ergun, V. Angelopoulos, J. Bonnell, and K.H. Glassmeier, Electric and magnetic field observations of Pc4 and Pc5 pulsations in the inner magnetosphere: A statistical study, J. Geophys. Res., 114, A12206, DOI: 10.1029/2009JA014243, 2009.

McPherron, R.L., Magnetic pulsations: their sources and relation to solar wind and geomagnetic activity, Surv. Geophys., 26, 545-592, DOI: 10.1007/s10712-005-1758-7, 2005.

Nicoll, K.A., and R.G. Harrison, Detection of lower tropospheric responses to solar energetic particles at mid-latitudes, Phys. Rev. Lett., 112, 225001, 2014.

Park, C.J., Downward mapping of high latitude ionospheric electric fields to the ground, J. Geophys. Res., 81, 168-174, DOI: 10.1029/JA081i001p00168, 1976.

Roble, R.G., and I. Tzur, The global atmospheric electrical circuit, National Academy Press, Washington, D.C., 1986.

Rycroft, M.J., and R.G. Harrison, Electromagnetic atmosphereplasma coupling: The global atmospheric electric circuit, Space Sci. Rev., 168, 363-384, DOI: $10.1007 / \mathrm{s} 11214-011-9830-8,2012$.

Sciffer, M.D., C.L. Waters, and F.W. Menk, Propagation of ULF waves through the ionosphere: inductive effect for oblique magnetic fields, Ann. Geophys., 22, 1155-1169, DOI: 10.5194/angeo-22-1155-2004, 2004.

Yair, Y., and B. Ziv, Introduction to Meteorology, Open University Press, Ra'anana, Israel, 2013.

Cite this article as: Elhalel G, Yair Y, Nicoll K, Price C, Reuveni Y \& Harrison RG: Influence of short-term solar disturbances on the fair weather conduction current. J. Space Weather Space Clim., 2014, 4, A26. 